\title{
First joint meeting of three European tuberculosis
}

\section{networks}

M J van der Werf (Marieke.vanderWerf@ecdc.europa.eu) ${ }^{1}$, C Erkens², A Gebhard², F Voitzwinkler³, M Dara ${ }^{4}$

1. European Centre for Disease Prevention and Control (ECDC), Stockholm, Sweden

2. KNCV Tuberculosis Foundation, The Hague, the Netherlands

3. Global Health Advocates, Brussels, Belgium

4. World Health Organization Regional Office for Europe, Copenhagen, Denmark

van der Werf M), Erkens C, Gebhard A, Voitzwinkler F, Dara M. First joint meeting of three European tuberculosis networks. Euro Surveill. 2013;18(37):pii=20583. Available online: http://www.eurosurveillance.org/ViewArticle.aspx?Articleld=20583

On 29 May 2013 three European tuberculosis (TB) networks met for the first time to discuss TB prevention, control and care in the World Health Organization (WHO) European Region including the European Union (EU). This meeting, which took place in The Hague, the Netherlands, provided a unique opportunity to discuss progress with the implementation of the Berlin Declaration on TB [1], the European Centre for Disease Prevention and Control (ECDC) Framework Action Plan to fight tuberculosis in the EU $[2,3]$, and the Consolidated Action Plan to prevent and combat multidrug- and extensively drug-resistant tuberculosis (M/ XDR-TB) in the WHO European Region [4]. Surveillance focal points, laboratory experts, and National TB Programme Managers (NTPs) exchanged lessons learned and discussed next steps to reach the targets defined in the plans.

To coordinate and improve TB prevention, control and care in the WHO European Region including the EU, professional networks have been established: (i) the Wolfheze movement aims to strengthen TB control in the WHO European Region [5]; the workshops of the Wolfheze movement provide a platform for NTPs, health authorities, laboratory experts, national TB surveillance correspondents, civil society organisations and other partners to discuss achievements, challenges and way forward; (ii) the European Tuberculosis Surveillance Network aims to improve the contribution of surveillance to TB control, and to promote standardised methods; (iii) the European Reference Laboratory Network for Tuberculosis (ERLN-TB) was established to strengthen TB diagnosis in the EU [6].

\section{Implementation of European TB}

prevention and control plans

Masoud Dara, WHO Regional Office Europe, Copenhagen, Denmark, and Marieke van der Werf, ECDC, Stockholm, Sweden, presented progress made with the implementation of the WHO European Region and EU TB prevention and control plans, respectively. They also presented the TB and MDR-TB epidemiological situation [7], the status of the indicators of the Berlin Declaration Monitoring and Evaluation Framework [7] and, finally, the epidemiological and core operational indicators of the Follow-up of the Framework Action Plan to fight tuberculosis in the EU [3] were assessed.

Since 2011, treatment of MDR-TB patients has been substantially scaled up with more than $97 \%$ of patients reported to be on treatment. However, treatment success rate of MDR-TB is far below the target of $75 \%$ envisaged for 2015. Furthermore there are frequent stockouts of second-line drugs for TB. The following key steps were presented to support further implementation of the Consolidated Action Plan:

- continued technical support to Member States;

- identify and address the social determinants of TB and M/XDR-TB;

- prepare a compendium of best practices;

- scale up best practices and patient-centred ambulatory care;

- strengthen country capacity in surveillance for producing reliable estimates of MDR-TB;

- introduce rational use of new TB medicines;

- develop interventions to move towards TB elimination in low TB incidence countries;

- define the role of surgery in TB and M/XDR-TB.

In the EU, only one of four epidemiological targets was met (i.e. overall decline of the five-year trend in TB case notification rates). Furthermore only one of five measured core targets was met (i.e. $100 \%$ of the national TB reference laboratories achieved a performance level of $180 \%$ for smear microscopy, culture and DST for firstand second-line drugs). It was therefore concluded that continued implementation of the Framework Action Plan to Fight Tuberculosis in the EU is needed.

After the general session the meeting focussed on four specific areas: (i) diagnosis; (ii) treatment and care; (iii) infection control, and; (iv) advocacy, partnerships and political commitment. First, selected low incidence and 
high TB priority countries presented their key achievements and challenges. This was followed by four working groups on the aforementioned themes.

\section{Diagnosis}

Gulnoz Uzakova, Global Fund to Fight AIDS, Tuberculosis and Malaria TB grant manager, Tashkent, Uzbekistan, considered the improved diagnostic infrastructure and implementation of an external quality assurance scheme (EQA) in Uzbekistan as key achievements. Also, rapid drug susceptibility tests are implemented both at central and peripheral level. Good political commitment, as well as partnership and collaboration between the national and international organisations have been established. However, the targets for culture confirmation, coverage of first-line drug sensitivity testing (DST), and coverage of second-line DST are not met. The logistics, such as transportation of sputum samples, need to be improved, as well as data interpretation and information flows.

In Italy, key achievements presented by Daniela Cirillo, San Raffaele Scientific Institute, Milan, include a wellfunctioning infrastructure of laboratories on three levels and implementation of EQA for both first and second line DST. There is also a good system for regular training of laboratory staff. The key challenges are the need to strengthen the surveillance system and data reporting within Italy and its regions and from the regional to the national level. There is also a lack of funds for the implementation and support of the regional and national laboratory networks.

The working group on diagnosis addressed several questions, among which the role of national TB reference laboratories, access to rapid TB diagnosis and existing problems in implementation of novel technologies and drug susceptibility testing to second line drugs. The main conclusions were that in principle the national reference laboratory is responsible for the quality of the TB laboratory network at country level. However, in practice not all national reference laboratories are able to perform this function because they are not authorised or because of funding. To ensure a high quality of laboratory test results, TB laboratories should be accredited by designated national bodies and/or have a quality management system. A challenge is that national legislations and regulations differ in the requirements and levels of laboratory accreditation. Universal access to rapid diagnosis of TB is currently hampered by the costs of the tests, administrative obstacles, lack of training and managerial capacity, and inappropriate requests of the tests by clinicians. The working group emphasised the need for a prioritisation of DST for fluoroquinolones (FQ) and injectable second line drugs to detect early XDR cases. Major challenges include the unavailability of laboratory supplies, the lack of EQA, high costs, a lack of standardisation and need to improve the clinical interpretation of test results.
Treatment and care

According to Armen Hayrapetyan National TB Programme, Yerevan, Armenia, the TB notification rate is steadily decreasing in Armenia. Since 2007, the treatment success of MDR-TB has remained low at about $53-55 \%$. Another challenge is the increasing number of individuals co-infected with TB and human immunodeficiency virus (HIV). Key achievements in Armenia are the availability of a TB/MDR/XDR response plan, and national guidelines for TB care. Also drugs for treatment of M/XDR-TB patients are now available. A programme for TB home-based care has been introduced. The key challenges for Armenia are the modernisation of the TB services, and the training of physicians, nurses and TB specialists.

In Hungary, there has been a steady decline of TB and the level of MDR-TB is low ( $13 \%$ ) as reported by Kovács Gábor, Koranyi National Institute for Tuberculosis and Pulmonology, Budapest. There is political commitment and a new national TB control plan is under development. Hungary has protocols for TB treatment and diagnosis which are in line with international recommendations. The country lacks adequate human resources in the healthcare sector in general and for TB specifically. There is a need to improve the model for treatment support to improve the treatment outcomes.

The working group on TB treatment and care discussed progress in improving universal access to TB treatment and care. Considerable progress has been made, especially by involving non-governmental organisations (NGOs) which link with vulnerable and high-risk groups. A key recommendation is to further increase the collaboration with NGOs, especially those working in HIV care. The availability of new drugs, such as bedaquiline, widens the treatment arsenal. Participants agreed that stringent pharmacovigilance, careful planning, ethical clearance and quality controls must be in place to ensure rational and effective use of new medicines. Countries are optimising their TB models of care, shifting from hospital based towards more ambulatory care, while improving quality of care and rational use of inpatient facilities with infection control standards. These need to be accompanied with training and retraining health staff in TB services and primary health care facilities. Another key recommendation is that a shift towards more ambulatory TB models of care should be accompanied by a reform of TB financing, well planned and intensive training of the healthcare workers involved and adequate psycho-social patient support throughout treatment.

\section{Infection control}

In Georgia, key achievements within the area of infection control as presented by Nestan Tukvadze, National Center for Tuberculosis and Lung Diseases, Tbilisi include the finalisation of national TB infection control guidelines in line with WHO recommendations, the implementation of a basic infection control risk assessment for TB service points, and the renovation of TB 
outpatient service points and microscopy laboratories. Currently Georgia is working on expanding the 'three I's' (intensified TB case-finding, initiate TB prevention with isoniazid preventive therapy and early antiretroviral therapy, and TB infection control [8]), training of healthcare workers and developing infection control standards for renovation and construction of healthcare facilities. Some of the key challenges that need to be addressed are monitoring and evaluation of implementation and impact of infection control measures, and adequate maintenance of engineering control measures.

Norway has comprehensive guidelines for TB control, care and prevention which are well disseminated and accepted by the healthcare providers according to Karin Rønning, Norwegian Institute of Public Health, Oslo. National infection control guidelines are available; however, they do not contain a separate TB infection control plan. The implementation of infection control measures is supported by comprehensive regulations and guidelines. The standards of healthcare facilities are generally very high, with adequate isolation capacity available. To stop TB transmission the challenge for Norway is to quickly identify cases and provide them with high quality treatment and care.

The working group discussed topics important for adequate TB infection control, such as national TB infection control guidelines and strategic plans, funding and other organisational mechanisms, human resources capacity building, and monitoring and evaluation of TB infection control activities.

The main conclusions were that progress has been made on TB infection control across the Region and that infection control should be an integral part of national strategic TB plans. Countries should focus on best possible (cost)-effective infection control measures.

Key recommendations included:

- ensure a proper funding mechanism for TB infection control measures;

- update regularly national TB infection control regulations;

- adapt TB infection control training modules based on country context;

- advocate for and develop plans for TB infection control operational research;

- develop country-adapted standardised specifications for ventilation systems, and for ultraviolet germicidal irradiation equipment.

Advocacy, partnerships and

political commitment

Jonathan Stillo, TB Europe Coalition, Bucharest, reported that Romania has a significant TB burden with a high number of MDR-TB cases and a treatment success rate for MDR-TB cases below the target. Frequent changes of high level decision-makers have resulted in a lack of coherence and continuity in TB control. There are serious challenges related to financing of TB control. A step towards strengthening advocacy and a push for political commitment was the formation of the Romanian Stop TB Partnership including 19 NGOs. The presence of the international community is important to build partnerships and to ensure political commitment.

Simon Logan, All-Party Parliamentary Group on Global Tuberculosis, London, United Kingdom, informed about this group which is working across political parties to address TB by arranging meetings in the parliament, working through parliamentary procedures such as debates, oral and written questions and publishing reports. Partnerships among NGOs, academics, civil servants and public health professionals are key to building a strong and united approach.

The working group gathered participants from civil society, national TB programmes and international organisations and aimed to identify the main challenges to advocacy in the region, what action could be taken to remedy these challenges and the tools and resources needed.

The main conclusions are that there is insufficient involvement of all stakeholders in advocacy and a general lack of understanding of its added value. Hence, the inadequacy of funds for advocacy activities, and the lack of capacity and expertise for civil society and others to run such activities.

Key recommendations were to document evidence of the added value of advocacy efforts, build the capacity of TB stakeholders in advocacy, enhance coordination and partnership at country level, increase political awareness of TB and advocate for more funding for advocacy.

\section{Conclusions}

The joint day finished with a presentation of the EU Standards for Tuberculosis Care [9]. This document describes the standards for four areas, i.e. TB diagnosis, TB treatment, HIV infection and comorbid conditions, public health and TB prevention (including infection control). Three of these had been discussed during the day as well.

This first joint meeting helped to facilitate exchange of good practices between professionals working in different areas of TB control and prevention in Europe. Key areas that need strenghtening to reach the targets of the TB control and prevention plans were identified and key recommendations were agreed on.

\section{Acknowledgements}

We acknowledge the contributions of Gulnoz Uzakova (Uzbekistan), Daniela Cirillo (Italy), Armen Hayrapetyan (Armenia), Kovács Gábor (Hungary), Nestan Tukvadze (Georgia), Karin Rønning (Norway), Jonathan Stillo (Romania), Simon Logan (UK), Kristin Kremer (WHO Regional Office for Europe), Francis Drobniewski (UK), Vladyslav Nikolayevskyy (UK), Vlad Furman (Kazachstan), Patrick Bertrand Belgium), leva Leimane (Netherlands), and Nina Bjerglund Andersen (WHO Regional Office for Europe). We especially would like 
to acknowledge Gerard de Vries (Netherlands), Barbara Hauer (Germany), Lena Fiebig (Germany), Andreas Sandgren (European Centre for Disease Prevention and Control), Martin van der Boom (WHO Regional Office for Europe), for their input for specific parts of the manuscript, and for reviewing the draft manuscript.

\section{Conflict of interest}

None declared.

Authors' contribution

Marieke J. van der Werf designed and drafted the manuscript and coordinated the input from the other authors. Connie Erkens provided input for specific parts of the manuscript, reviewed the draft manuscript, and gave final approval of the version to be published. Agnes Gebhard provided input for specific parts of the manuscript, reviewed the draft manuscript, and gave final approval of the version to be published. Fanny Voitzwinkler provided input for specific parts of the manuscript, reviewed the draft manuscript, and gave final approval of the version to be published. Masoud Dara provided input for specific parts of the manuscript, reviewed the draft manuscript, and gave final approval of the version to be published.

\section{References}

1. World Health Organization (WHO). The Berlin Declaration on Tuberculosis. Berlin: World Health Organization Regional Office for Europe and All Against Tuberculosis. Copenhagen: WHO; 2007. Available from: http://www.euro.who.int/_data/assets/ pdf_file/oo08/68795/E91369.pdf

2. European Centre for Disease Prevention and Control (ECDC). Framework action plan to fight tuberculosis in the European Union. Stockholm: ECDC; 2008. Available from: http://ecdc. europa.eu/en/publications/publications/0803_spr_tb_action_ plan.pdf

3. European Centre for Disease Prevention and Control (ECDC). Progressing towards TB elimination. Stockholm: ECDC; 2010. Available from: http://ecdc.europa.eu/en/publications/ publications/101111_spr_progressing_towards_tb_elimination. pdf

4. World Health Organization (WHO) Regional Office for Europe. Roadmap to prevent and combat drug-resistant tuberculosis. Copenhagen, WHO Regional Office for Europe; 2011. Available from: http://www.euro.who.int/_data/assets/pdf file/0014/152015/e95786.pdf

5. Veen J, Migliori GB, Raviglione M, Rieder HL, Dara M, Falzon D, et al. Harmonisation of TB control in the WHO European region: the history of the Wolfheze Workshops. Eur Respir J. 2011 Apr;37(4):950-9. http://dx.doi. org/10.1183/09031936.00019410. PMid:20530031.

6. Drobniewski FA, Nikolayevskyy V, Hoffner S, Pogoryelova O, Manissero D, Ozin AJ. The added value of a European Union tuberculosis reference laboratory network--analysis of the national reference laboratory activities. Euro Surveill. 2008 Mar 18;13(12): pii=8076. Available from: http://www. eurosurveillance.org/ViewArticle.aspx?Articleld=8076. PMid:18761994.

7. European Centre for Disease Prevention and Control (ECDC)/ World Health Organization (WHO) Regional Office for Europe. Tuberculosis surveillance and monitoring in Europe, 2013. Stockholm/Copenhagen: ECDC / WHO Regional Office for Europe; 2013. Available from: http://www.ecdc.europa.eu/ en/publications/Publications/Tuberculosis-surveillancemonitoring-2013.pdf

8. World Health Organization (WHO). WHO policy on collaborative TB/HIV activities: Guidelines for national programmes and other stakeholders. Geneva: WHO; 2012. Available from: http://whqlibdoc.who.int/publications/2012/9789241503006_ eng.pdf

9. Migliori GB, Zellweger JP, Abubakar I, Ibraim E, Caminero JA, De Vries G, et al. European union standards for tuberculosis care. Eur Respir J. 2012 Apr;39(4):807-19. http:// dx.doi.org/10.1183/09031936.00203811. PMid:22467723. PMCid:PMC3393116. 